\title{
Development of electrochemical biosensors based on sol-gel enzyme encapsulation and protective polymer membranes
}

\author{
Rasa Pauliukaite • Monika Schoenleber • \\ Pankaj Vadgama $\cdot$ Christopher M. A. Brett
}

Received: 2 August 2007 /Revised: 26 October 2007 / Accepted: 14 November 2007 / Published online: 16 December 2007

(C) Springer-Verlag 2007

\begin{abstract}
Protective polymer coatings have been used to enhance the retention of enzymes in sol-gel films as immobilisation phases in electrochemical biosensors. Carbon film electrodes were electrochemically modified with poly(neutral red) (PNR). These electrodes were coated with oxysilane sol-gels incorporating glucose oxidase and an outer coating of carboxylated PVC (CPVC) or polyurethane (PU), with and without Aliquat-336 or isopropyl myristate (IPM) plasticizer, was applied. The biosensors were characterised electrochemically using cyclic voltammetry and amperometry, electrochemical impedance spectroscopy and scanning electron microscopy. Impedance spectra showed that the electrode surface is most active when the sol-gel-GOx layer is not covered with a membrane. However, membranes without plasticizer extend the lifetime of the biosensor to more than 2 months when PU is used as an outer membrane. The linear range of the biosensors was found to be $0.05-0.50 \mathrm{mM}$ of glucose and the biosensor with PU outer membrane exhibited higher sensitivity (ca.117 $\mathrm{nA} \mathrm{mM}^{-1}$ ) in the region of linear response than that with CPVC. The biosensors were applied to glucose measurement in natural samples of commercial orange juice.
\end{abstract}

\footnotetext{
R. Pauliukaite C. M. A. Brett $(\triangle)$

Departamento de Química, Universidade de Coimbra, 3004-535 Coimbra, Portugal

e-mail: brett@ci.uc.pt

M. Schoenleber $\cdot$ P. Vadgama

IRC in Biomedical Materials, Queen Mary University of London,

Mile End Road,

London E1 4NS, UK
}

Keywords Carbon film electrode · Poly(neutral red) . Biosensor - Amperometry Cyclic voltammetry . Electrochemical impedance spectroscopy. Glucose determination Polymer membrane

\section{Introduction}

The sol-gel technique is often used for the encapsulation of bioactive substances, particularly enzymes, in the development of biosensors [1-5]. Organic-inorganic silanes polymerised by the sol-gel method are attractive materials since they combine, in a single phase, both the properties of the rigid three-dimensional porous silica network and the particular chemical reactivity of the organic components [4]. Some parameters, such as $\mathrm{pH}$, gelation time, transparency and hydrophobicity can be adapted to encapsulate labile protein molecules such as enzymes [1-3, 5]. Usually the sol-gel is formed from the oxysilane in a two-step reaction: hydrolysis, and condensation, which is responsible for building the $\mathrm{SiO}_{2}$ matrix within which enzyme molecules can be encapsulated [3].

Sol-gel enzyme encapsulation has been applied to different electrochemical [3, 6-15] and optical [16, 17] biosensors in the last few years. However, instability problems in some electrochemical biosensors have been reported [8, 13-15], which could be due to a small amount of enzyme leaching or to its inactivation. In order to solve this problem polymer membranes, particularly polyurethane (PU), were applied as an outer protecting layer [15].

Polymer membranes have been widely used for protein retention for over two decades [18-20]. Different nonsol-gel membranes have been used for this purpose, including poly(vinyl chloride) (PVC), different polyacry- 
lates and polyurethane [19-23]. For example, membrane constructs can be created with incorporated protein/enzyme molecules by cross-linking using amine functional groups $[19,24]$, or a discrete protective layer can be formed over an enzyme layer [25]. The polymers should be chosen according to the conditions under which the sensor would operate, e.g. buffered samples, natural biological samples, in vivo, in vitro, etc. Modified PVC with carboxyl groups, carboxylated PVC (CPVC), has been applied to biosensor preparation by the covalent bonding of alcohol oxidase [26] or urease $[27,28]$.

Different electrode materials have been employed for biosensor preparation; however, carbon-based electrodes are most widely used for this purpose. Carbon film electrodes, obtained by coating a ceramic substrate with a thin deposit of pyrolytic carbon [29-31], have been successfully utilised. They have been used for developing glucose biosensors without mediators [32] but, in order to improve selectivity, biosensors have been modified with redox mediators, particularly copper [13] or cobalt [33] hexacyanoferrates, methyl viologen [34] and poly(neutral red) [35].

In previous studies $[13,14]$, it was reported that sol-gel enzyme electrodes can lead to unsatisfactorily short lifetimes, partly due to enzyme leaching. Therefore, in this work several polymer membranes, particularly polyurethane and carboxylated poly(vinyl chloride), were applied over the sol-gel-enzyme layer to prevent enzyme leaching. The results of glucose assay in relation to sensitivity, stability and response time in standard solutions obtained at sol-gel-enzyme-polymer membrane biosensors are presented.

\section{Experimental}

\section{Chemicals and solutions}

3-Glycidoxypropyltrimethoxysilane (GOPMOS), methyltrimethoxysilane (MTMOS) and neutral red $\left(N^{8}, N^{8}, 3\right.$-trimethylphenazine-2,8-diamine), and Aliquat-336 were obtained from Aldrich (Germany) (structures of these compounds are presented in Table 1). Glucose oxidase (GOx) from Asperigillus niger (EC 1.1.3.4) and anhydrous $\alpha$ - $\mathrm{D}(+)$-glucose crystals were from Sigma (Germany). Carboxylated poly (vinyl chloride) and isopropyl myristate (IPM; see structure in Table 1) were purchased from Fluka (Switzerland), and polyurethane prepolymer was a kind gift from Baxenden (UK). Tetrahydrofuran (THF) was obtained from Riedel-de Haën (Germany). All products were analytical grade and were used without any further purification.

Electrolyte solution, $0.1 \mathrm{M}$ phosphate buffer saline (PBS), $\mathrm{pH} 7.0$, was prepared from sodium dihydrogen- phosphate and disodium hydrogenphosphate (Riedel-de Haën, Germany), to which $0.05 \mathrm{M} \mathrm{NaCl}$ was added. Millipore Milli-Q nanopure water (resistivity $>18 \mathrm{M} \Omega \mathrm{cm}$ ) was used for the preparation of all solutions. Experiments were performed at room temperature, $25 \pm 1{ }^{\circ} \mathrm{C}$.

\section{Biosensor preparation}

Electrodes were made from carbon film resistors of ca. $2-\Omega$ nominal resistance, as described elsewhere [29-31]. The exposed electrode geometric area was ca. $0.20 \mathrm{~cm}^{2}$. The electrodes were electrochemically pretreated prior to electropolymerisation with neutral red by cycling between -1.0 and $+1.0 \mathrm{~V}$ vs. saturated calomel electrode (SCE) in deoxygenated $0.1 \mathrm{M} \mathrm{KNO}_{3}$ solution for not less than ten cycles, until stable cyclic voltammograms were obtained.

Poly(neutral red) (PNR) was polymerised on carbon film electrodes by electropolymerisation from a $1 \mathrm{mM}$ solution of its monomer (neutral red, structure in Table 1) in aqueous $0.05 \mathrm{M}$ phosphate buffer, $\mathrm{pH} 5.5$, and $0.1 \mathrm{M}$ $\mathrm{KNO}_{3}$ solution, by cycling the applied potential from -1.0 to $1.0 \mathrm{~V}$ vs. SCE 15 times at a potential sweep rate of $50 \mathrm{mV} \mathrm{s}^{-1}$. The electrode was then rinsed with deionised water to remove free monomer, after which it was left to dry for 1 day in air at room temperature.

Sol-gel solution was prepared by mixing the two oxysilanes with water in optimised ratios [13, 14]: GOPMOS/ MTMOS/ $\mathrm{H}_{2} \mathrm{O}$ 130:70:600 $\mu \mathrm{L}$, respectively. A 2- $\mu \mathrm{L}$ aliquot of $6 \mathrm{M} \mathrm{HCl}$ solution was added to the mixture obtained to accelerate hydrolysis of the oxysilanes. The mixture obtained was intensively stirred for a few minutes and then sonicated for $15 \mathrm{~min}$. The solution was then heated in a hot air stream (ca. $70{ }^{\circ} \mathrm{C}$ ) to evaporate the alcohol formed during hydrolysis of the oxysilanes [36] until the mixture lost $40 \%$ of its volume. It was then left for $1 \mathrm{~h}$ at room temperature to cool down and neutralized to $\mathrm{pH} 7.0$ with $0.1 \mathrm{M} \mathrm{NaOH}$ solution. A $50-\mu \mathrm{L}$ aliquot of this solution was carefully mixed with $15 \mu \mathrm{L}$ of GOx $(10 \%)$ solution in $0.1 \mathrm{M}$ PBS solution $\mathrm{pH} 7.0$ and left for $1 \mathrm{~h}$ to equilibrate. The PNR-coated carbon film electrode was then immersed in sol-gel-enzyme solution for $5 \mathrm{~min}$, removed and left to form a xerogel at $4{ }^{\circ} \mathrm{C}$ for 2 days.

Membrane solutions were prepared by dissolving either CPVC or PU in THF by sonication of the polymer-solvent mixture until transparent solutions were obtained. The optimised composition for polymer preparation was 3.5\% (w/v) CPVC or $20 \%$ PU (v/v). Some plasticizers, $5 \%(\mathrm{v} / \mathrm{v})$ Aliquat-336 or $1.6 \%(\mathrm{v} / \mathrm{v})$ IPM, were added to the solutions to improve membrane properties. The mixtures were sonicated once again to obtain homogeneous solutions. Six different membrane solutions were used: (1) $3.5 \%$ CPVC, (2) $3.5 \%$ CPVC $+5 \%$ Aliquat-336, (3) $3.5 \%$ CPVC $+1.6 \%$ IPM, (4) $20 \% \mathrm{PU}$, (5) $20 \% \mathrm{PU}+5 \%$ Aliquat-336 and (6) $20 \% \mathrm{PU}+$ 
Table 1 Structures of organic compounds used for biosensor preparation

\section{Compound}

Structure

N8,N8,3-trimethylphenazine-2,8-

diamine (Neutral red monomer)

3-Glycidoxypropyltrimethoxysilane

(GOPMOS)<smiles>Cc1cc2nc3ccc(N(C)C)cc3nc2cc1N</smiles><smiles>CO[Si](CCCOCC1CO1)(OC)OC</smiles>

Methyltrimethoxysilane

(MTMOS)<smiles>CO[Si](C)(OC)OC</smiles>

Carboxylated polyvinyl chloride<smiles>CC(C)(C)CCCl</smiles>

Polyurethane<smiles>CCCCC(C)NC(=O)OCC</smiles>

Aliquat-336

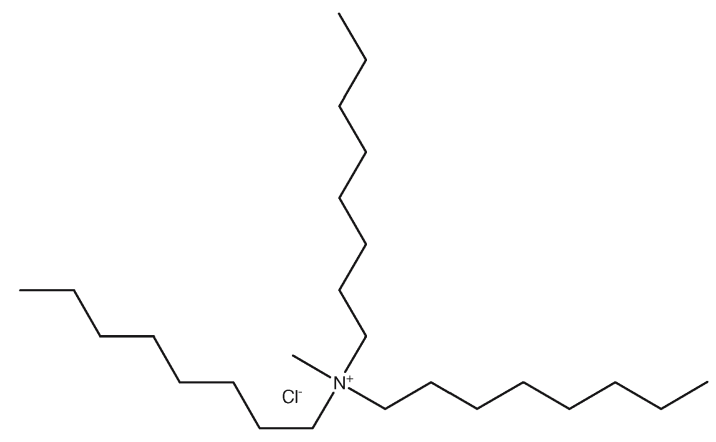

Isopropyl myristate (IPM)

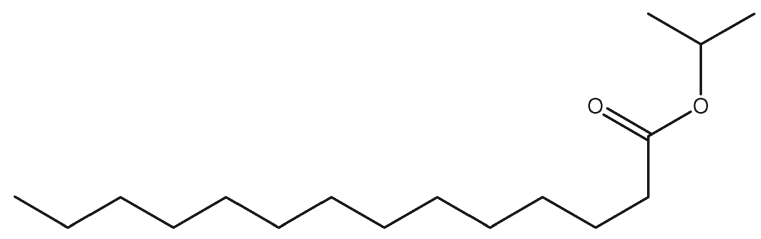


$1.6 \%$ IPM. The electrodes with the formed sol-gel layer (after 2 days) were immersed in one of the membrane solutions for a few seconds and left in air for $30 \mathrm{~min}$ for solvent evaporation to leave a thin outer membrane layer.

Prepared biosensor assemblies were stored at $4{ }^{\circ} \mathrm{C}$ in PBS solution when not in use.

Methods and instruments

Cyclic voltammetry (CV) and amperometric measurements were performed in a three-electrode electrochemical cell containing a sol-gel encapsulated enzyme carbon film working electrode, a platinum foil as counter electrode and a saturated calomel electrode (SCE) as reference. Measurements were performed using a computer-controlled $\mu$-Autolab Type II potentiostat/galvanostat with GPES 4.9 software (Eco Chemie, Netherlands).

Electrochemical impedance spectroscopy (EIS) measurements were carried out in the same electrochemical cell as described above with a PC-controlled Solartron 1250 frequency response analyser coupled to a Solartron 1286 electrochemical interface using ZPlot 2.4 software (Solartron Analytical, UK). The frequency was scanned from $65 \mathrm{kHz}$ to $0.1 \mathrm{~Hz}$ with ten points per frequency decade, with a sinusoidal voltage perturbation amplitude of $10 \mathrm{mV}$, auto-integration time $60 \mathrm{~s}$, with short accuracy mode, at $90 \%$ significance level. Fitting to electrical equivalent circuits was performed with ZView 2.4 software (Scribner Associates, USA).

Scanning electron microscopy investigations were performed using a JEOL JSM 6300 scanning electron microscope after the specimens had been plated with gold under vacuum.

Independent analysis of glucose concentrations in commercial samples was done using a spectrophotometric enzyme assay kits (Cat 0139 106, Boehringer, Mannheim) and analysis at $340 \mathrm{~nm}$ using a SPECORD S100 (Analytik Jena GmbH, Jena, Germany) UV-VIS spectrophotometer running Aspect Plus Version 1.5 software.

\section{Results and discussion}

Electrochemical and surface characterisation of sol-gel/membrane biosensors

In order to follow changes in the electrochemical behaviour of the sol-gel/membrane-based biosensor during its preparation, cyclic voltammograms (CVs) and impedance spectra were recorded after each step of electrode preparation in 0.1 M PBS solution, $\mathrm{pH}$ 7.0. The results obtained are presented and described in this section.

The sol-gel was prepared from a GOPMOS and MTMOS mixture, volume ratio 2:1 in water and with the addition of $\mathrm{HCl}$ as catalyst (see Experimental). The composition of the outer polymer coating was also optimised via the response to glucose in PBS solution. Table 2 presents results from optimisation of the polymer concentration for the preparation of CPVC and PU membrane coatings without plasticizer. The best concentrations were found to be 3.5 and $20 \%(\mathrm{w} / \mathrm{v})$ for CPVC and $\mathrm{PU}$, respectively, in THF. These concentrations were used for further biosensor characterisation and application. Membranes made from more concentrated polymer solutions did not allow analyte to pass through to the sol-gel layer sufficiently easily for reaction with the enzyme so amperometric response was attenuated. For too low concentrations of polymer solution the results suggest that uniform membranes were probably not formed, see Table 2 . However, there is a report of the use of a PU membrane applied over the sol-gel layer for glucose microsensors made using a lower polymer concentration, and the sensor response was sufficient; however, here PU was blended with a hydrophilically modified PU in the weight ratio $7: 3$ [15].

\section{Cyclic voltammetry}

CVs were registered in PBS solution in the potential range from -1.0 to $+1.0 \mathrm{~V}$ vs. SCE. Neutral red polymerisation has been described in detail in previous work $[14,35]$ and in the Experimental. The PNR film was left to stabilise for 1 day, and before sol-gel deposition, CVs were registered in 0.1 M PBS pH 7.0 (see Fig. 1 dotted line). The electrochemical behaviour of PNR strongly depends on $\mathrm{pH}$ and well-defined polymer peaks are obtained at $\mathrm{pH}$ 4-6 $[37,38]$. However, the polymer peaks are broader and the peak current is lower at neutral pH, as is seen in Fig. 1. The

Table 2 Optimisation of CPVC and PU concentration for membrane preparation according to $\mathrm{PNR} /$ sol-gel-GOx/membrane electrode amperometric response to $200 \mu \mathrm{M}$ of glucose in $0.1 \mathrm{M} \mathrm{PBS}, \mathrm{pH} 7.0$ at $-0.25 \mathrm{~V}$ vs. SCE

\begin{tabular}{ll}
\hline Polymer concentration in THF $(\%)$ & Response to glucose (nA) \\
\hline CPVC & \\
4.8 & 10 \\
3.5 & 15 \\
3.0 & 13 \\
2.5 & 9 \\
PU & \\
50 & 5 \\
40 & 10 \\
30 & 25 \\
20 & 37 \\
10 & 20 \\
\hline
\end{tabular}




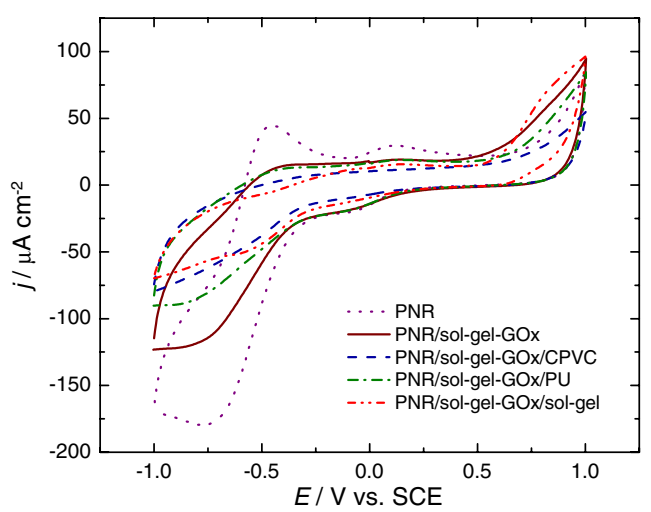

Fig. 1 Cyclic voltammograms at carbon film electrodes modified by PNR ( ...), PNR/sol-gel and PNR/sol-gel-GOx (-), PNR/sol-gelGOx/CPVC (-..), PNR/sol-gel-GOx/PU ( -...), PNR/sol-gelGOx/sol-gel ( -..-). Supporting electrolyte 0.1 M PBS, pH 7.0. Potential scan rate $50 \mathrm{mV} \mathrm{s}^{-1}$

monomer oxidation peak, usually present at potentials between +0.8 and $+0.9 \mathrm{~V}$ vs. SCE, is shifted to a significantly more positive potential and is out of the potential range studied.

Cyclic voltammograms were recorded on PNR-modified electrodes, after deposition of the sol-gel layer with encapsulated enzyme and with and without outer coatings (Fig. 1). As expected, the sol-gel acts as a diffusion membrane for counterions and the Faradaic current corresponding to PNR decreases significantly (Fig. 1, solid line). Addition of enzyme hardly changes the electrochemical behaviour of the biosensor, since the enzyme is electrochemically inactive and any co-factor concentration is too small to make changes to the CV of poly(neutral red). All the other CVs with any type of membrane presented in Fig. 1 are with entrapped enzyme. It is interesting that with a superimposed sol-gel layer an oxidation wave again appears at approximately $+0.8 \mathrm{~V}$ vs. SCE, where there is usually irreversible oxidation of neutral red monomer. This wave is seen with or without enzyme and so does not appear to be caused by the biosensing component itself. The mediated system here is quite distinct from those where the sol-gel-immobilised oxidase generates an indirect response via $\mathrm{H}_{2} \mathrm{O}_{2}$ [39].

After application of an outer protecting membrane of CPVC or PU (Fig. 1, dashed and dash-dot-dash lines, respectively), the PNR peak current again decreases and it is difficult to distinguish the peaks; the oxidation wave at $+0.8 \mathrm{~V}$ also disappears. These CVs were recorded using overlying membranes from solutions of $3.5 \%$ CPVC in THF (w:v) and 20\% PU in THF (v:v), optimised with respect to the response signal to glucose (see above).

As an alternative, a second sol-gel layer (without enzyme) was also deposited as an outer membrane coating on top of the first layer with enzyme. In this case, the CV recorded in PBS is rather similar to one with CPVC (Fig. 1, dash-dot-dot-dash curve). However, PNR redox peaks are still lower than at the biosensor with CPVC and there is a quite well defined oxidation wave at $+0.8 \mathrm{~V}$, which suggests that this oxidation wave comes from some electrochemical changes in the sol-gel itself.

Usually plasticizers are used to improve the physical properties of a polymer material [18-20]; Aliquat-336 and isopropyl myristate (see structure in Table 1) were used as plasticizers in this work to manipulate permeability. The influence of the plasticizers on the voltammetric behaviour of the biosensors is shown in Fig. 2. In the case of CPVC, plasticizers have a greater influence on the electrochemical behaviour of the biosensor, especially IPM (Fig. 2a, dashdot-dash line). This probably occurs due to the lower CPVC concentration compared with PU. Addition of 5\% Aliquat to the CPVC membrane composition led to higher reduction peak currents in the potential region between -0.6 and $-1.0 \mathrm{~V}$, i.e. in the region of PNR reduction, as well as in the monomer oxidation region, from +0.7 to +1.0 V, (Fig. 2a, dashed line) compared with CPVC without any plasticizer. These effects are even more marked when IPM is used as plasticizer; however, oxidation of PNR is also significantly suppressed. This suggests enhanced glucose transport through plasticized membranes, which
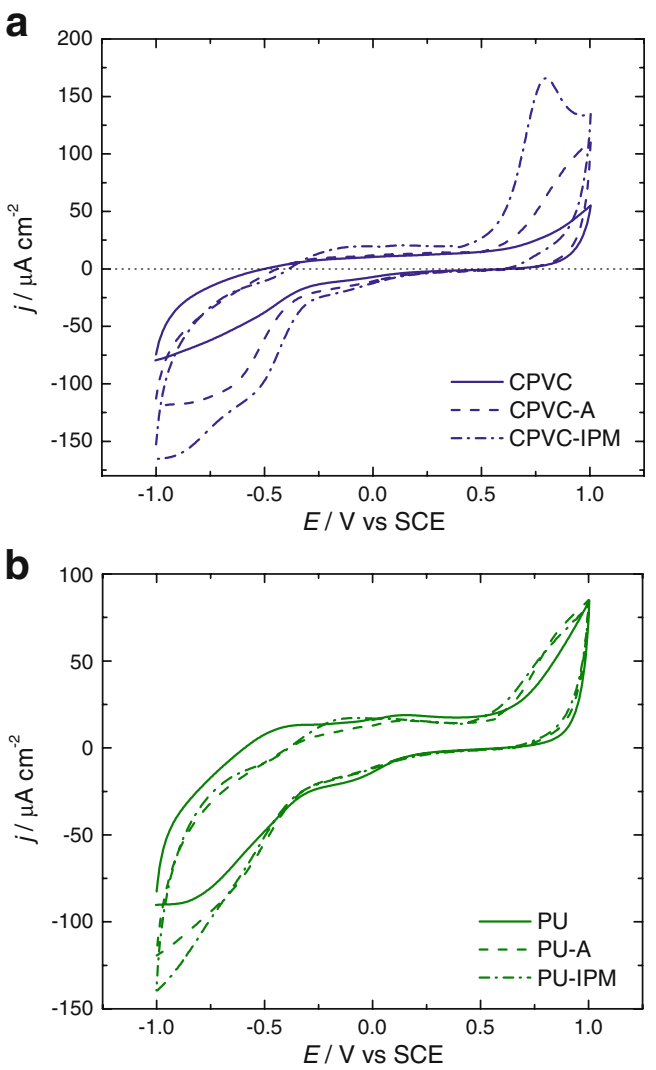

Fig. 2 Cyclic voltammograms at a PNR/sol-gel-GOx/CPVC and b $\mathrm{PNR} /$ sol-gel-GOx/PU without plasticizer, and with Aliquat (A) or IPM as plasticizer. All other conditions as in Fig. 1 
was certainly expected with the hydrophilic surfactant, thought not with the non-polar IPM. The effect of IPMenhanced transport could have been the result of structural (depth, porosity) changes to the formed membrane. Similar behaviour was observed at the biosensor with a PU outer membrane except that in this case, due to the higher polymer concentration, there was not such a large difference between PU with and without plasticizer (Fig. 2b).

\section{Electrochemical impedance spectroscopy}

Impedance spectra were recorded after each biosensor preparation step in the same way as in cyclic voltammetry, i.e. after electropolymerisation of neutral red; sol-gel deposition with and without enzyme; and outer membrane deposition. The sol-gel layer without enzyme was deposited to study any influence from the presence of enzyme on the electrode interfacial characteristics. Impedance spectra are presented in Fig. 3 which shows both complex plane and Bode plots. Spectra were recorded at three different potentials: at $0.0 \mathrm{~V}$, where slow protonation of ionogenic groups occurs; at $-0.25 \mathrm{~V}$, the chosen operating potential; and at $-0.5 \mathrm{~V}$, where the redox process of PNR takes place.

Impedance spectra in the complex plane plots at $0.0 \mathrm{~V}$ remain similar in form after each step in biosensor preparation - only the magnitude of the impedance varies.
Fig. 3 Complex plane (left) and Bode plots (right) at PNRmodified carbon film electrodes (open circles), with sol-gel layer without enzyme (red circles), with GOx immobilised without any protecting membrane (turquoise squares) and with CPVC (blue triangles) or PU (green triangles). Spectra were recorded at a $0.0 \mathrm{~V}, \mathbf{b}-0.25 \mathrm{~V}$ and $\mathbf{c}-0.50 \mathrm{~V}$ vs. SCE
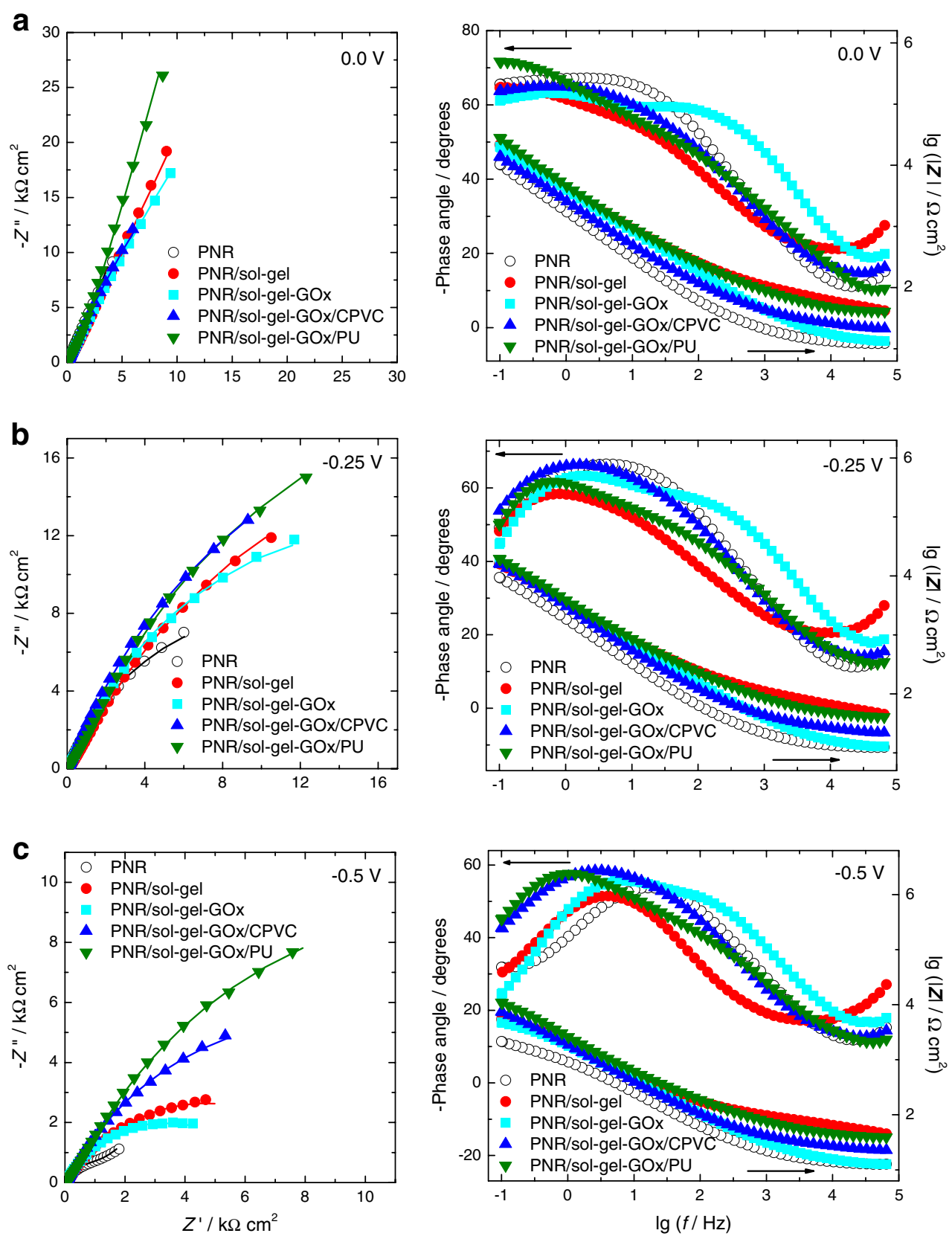
The linear nature of the spectra indicates charge separation over the whole frequency region. At $-0.25 \mathrm{~V}$, impedance values are lower and spectra curve in the low frequency region due to the oxidation of PNR. At $-0.5 \mathrm{~V}$, closer to the formal potential of the PNR redox couple, impedance values again decrease and the shapes of the spectra depend to a greater extent on the exact biosensor assembly in contrast to the other potentials. Uncoated PNR films show a more complex behaviour at this potential compared with coated ones, since, as is seen from Fig. 1, there are significantly higher PNR redox peaks than at sol-gel-coated PNR mediator films under these conditions. Differences in the shape of the spectra are seen between PNR/sol-gel and PNR/sol-gel-GOx electrodes which suggests that the enzyme changes the sol-gel morphology and structure as was found using AFM in sol-gel morphology studies [40].

Usually sol-gel and polyurethane films are investigated by EIS in the context of corrosion protection, so that spectra are recorded at the open circuit potential [41, 42]. The spectra from the complex plane and Bode plots at solgel-coated metals are rather similar those in this study at $-0.25 \mathrm{~V}$ vs. SCE in Fig. 3 and depend significantly on the identity of the substrate material (metal or alloy) [41]. Spectra of polyurethane films on stainless steel at $+0.1 \mathrm{~V}$ vs. SCE are different from those at the biosensor investigated here: there was mass transport control over the whole frequency range [42]. These facts demonstrate that the film structure can depend strongly on the electrode substrate and modifying layers.

In order to describe the processes occurring at the surface of PNR, an equivalent circuit model was used for fitting all spectra, consisting of the cell resistance, $R_{\Omega}$, in series with a parallel combination of a charge-transfer resistance, $R_{\mathrm{ct}}$ and a constant phase element, CPE, modelled as a non-ideal capacitance, according to [43]

$\mathrm{CPE}=1 /(C i \omega)^{\alpha}$

where $C$ is capacitance in $\mu \mathrm{F} \mathrm{cm}{ }^{-2} \mathrm{~s}^{\alpha-1}, \omega$ is the frequency in radians, and $\alpha$ represents surface non-uniformity, being equal to unity if the surface is smooth and uniform. This model was combined in series with a resistance, $R_{\mathrm{f}}$, and another CPE to represent the electrical properties of all the extra film components, when PNR was covered with solgel or sol-gel-enzyme layer and with the outer membrane layer. Table 3 gives the parameters deduced from the first $R$-CPE couple representing the interfacial processes and corresponding to the fitting of the high frequency part of the spectra. Values of $R_{\Omega}$ vary from 11 to $15 \Omega \mathrm{cm}^{2}$, except at PNR/sol-gel-GOx/PU, where $R_{\Omega}$ was ca. $32 \Omega \mathrm{cm}^{2}$. The charge-transfer resistance decreases with increasingly negative potential due to electrochemical reactions of the PNR [34-36]. An exception is PNR/sol-gel-GOx, demonstrating evidence of an additional reaction which is going on at this electrode as is also easy to identify from the Bode plot (Fig. 3). The $\alpha$ values vary from 0.7 to 1.0 and depend on the electrode composition: $\alpha$ is closest to 1.0 at the PNR/ sol-gel-GOx electrode which indicates that the surface is almost homogeneous and uniform.

\section{Scanning electron microscopy}

Figure 4 presents the SEM images at carbon film electrodes coated in various ways. All electrodes were coated with PNR film and sol-gel layer and some of them had GOx entrapped into the sol-gel layer and an outer polymer membrane without any plasticizer. The sol-gel layer seems
Table 3 Analysis of the electrochemical impedance data at carbon film electrodes

\begin{tabular}{lllll}
\hline$E$ vs. SCE (V) & $\begin{array}{l}\text { C film electrode } \\
\text { coating }\end{array}$ & $R_{\mathrm{ct}}\left(\mathrm{k} \Omega \mathrm{cm}^{2}\right)$ & $\begin{array}{l}C \\
\left(\mu \mathrm{F} \mathrm{cm} \mathrm{c}^{-2} \mathrm{~s}^{\alpha-1}\right)\end{array}$ & \\
\hline 0.0 & & $73.6 \pm 4.4$ & $0.81 \pm 0.01$ \\
& PNR & $193 \pm 8$ & $67.7 \pm 3.6$ & $0.73 \pm 0.01$ \\
& + sol-gel & $437 \pm 13$ & $533.7 \pm 7.9$ & $1.00 \pm 0.01$ \\
& +sol-gel-GOx & $7 \pm 1$ & $99.9 \pm 5.3$ & $0.76 \pm 0.01$ \\
& + CPVC & $153 \pm 7$ & $54.3 \pm 3.8$ & $0.84 \pm 0.01$ \\
& + PU & $524 \pm 15$ & $57.9 \pm 5.1$ & $0.83 \pm 0.01$ \\
& PNR & $56 \pm 3$ & $78.8 \pm 7.2$ & $0.75 \pm 0.01$ \\
& + sol-gel & $49 \pm 3$ & $137.6 \pm 6.0$ & $0.94 \pm 0.01$ \\
& +sol-gel-GOx & $10 \pm 1$ & $77.7 \pm 6.0$ & $0.79 \pm 0.01$ \\
& + CPVC & $51 \pm 2$ & $89.0 \pm 3.3$ & $0.88 \pm 0.01$ \\
& + PU & $33 \pm 2$ & $114.9 \pm 7.1$ & $1.00 \pm 0.01$ \\
& PNR & $1 \pm 0$ & $133.6 \pm 6.7$ & $0.75 \pm 0.01$ \\
& + sol-gel & $9 \pm 1$ & $157.8 \pm 7.0$ & $0.77 \pm 0.01$ \\
& + sol-gel-GOx & $5 \pm 0$ & $141.4 \pm 1.0$ & $0.71 \pm 0.01$ \\
& + CPVC & $17 \pm 1$ & $135.9 \pm 8.1$ & $0.84 \pm 0.01$ \\
\hline & + PU & $15 \pm 1$ &
\end{tabular}


Fig. 4 SEM images of PNR film polymerised on carbon film electrodes covered with a sol-gel, b sol-gel-entrapped GOx, c sol-gel-entrapped GOx with outer membrane of CPVC, and d sol-gel-entrapped GOx with outer membrane of PU
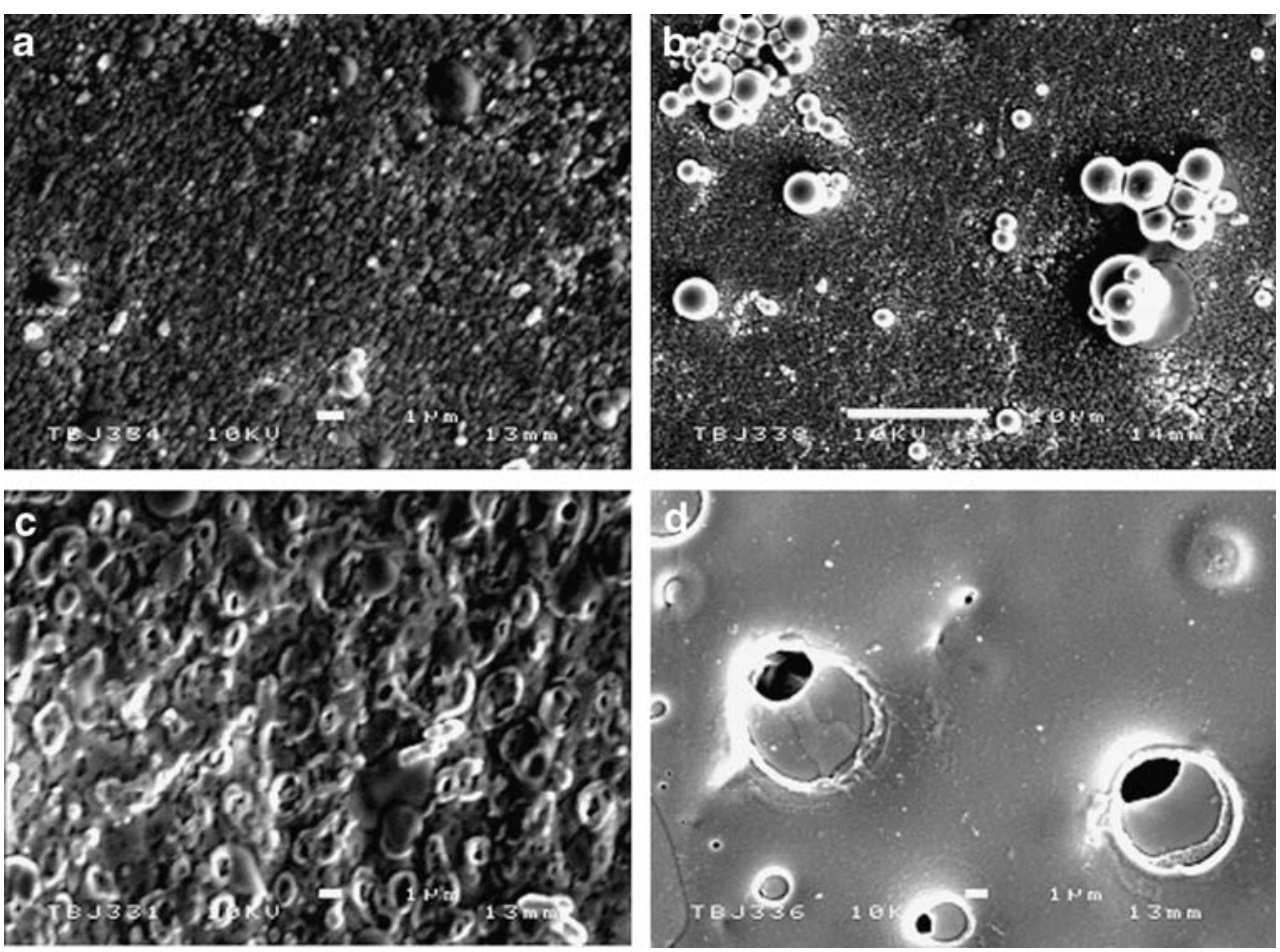

to be rather flat and homogeneous (Fig. 4a) as would be expected from a glass-like xerogel layer. Interestingly, after GOx entrapment the sol-gel layer is no longer homogeneous and some clusters are visible at the surface (Fig. 4b). These clusters are possibly due to enzyme aggregates. Similar changes in the sol-gel layer after enzyme entrapment have been found using AFM [40].

After application of the outer polymer membrane the surface topography is different and depends on the nature of the polymer. When CPVC was used as a protective membrane the electrode surface was rather homogeneous but not perfectly flat, showing onion-ring-shaped structure (Fig. 4c). The other polymer, PU, formed a much more uniform and flat surface, although a few islands of discrete defects are seen, as highlighted in Fig. 4d. These results agree with those obtained using electrochemical methods and can explain the response and stability of the biosensors prepared with these polymer membranes (see below).

\section{Response to the analyte}

The biosensors with the outer diffusion membranes were

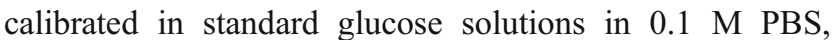
$\mathrm{pH}$ 7.0. Calibration data were analysed and the main analytical parameters calculated. The biosensors were also checked for interference from some sugars, organic acids and phenol. Additionally, their lifetime and storage stability over a period of 70 days was examined. The electrodes were stored at $+4{ }^{\circ} \mathrm{C}$ in PBS solution when not in use.

\section{Calibration of the biosensors}

Calibration curves were recorded at biosensors without an outer membrane, and at biosensors with CPVC and PU outer diffusion membranes with and without plasticizers (Aliquat-336 and IPM). Application of the diffusion membrane decreased the current response to glucose but it still remained acceptable for glucose measurement, as shown in Fig. 5, except in cases when Aliquat was used as plasticizer where a marked loss in response was observed during the assay; this is a possible result of enzyme loss induced by the hydrophilic surfactant. When PU was used

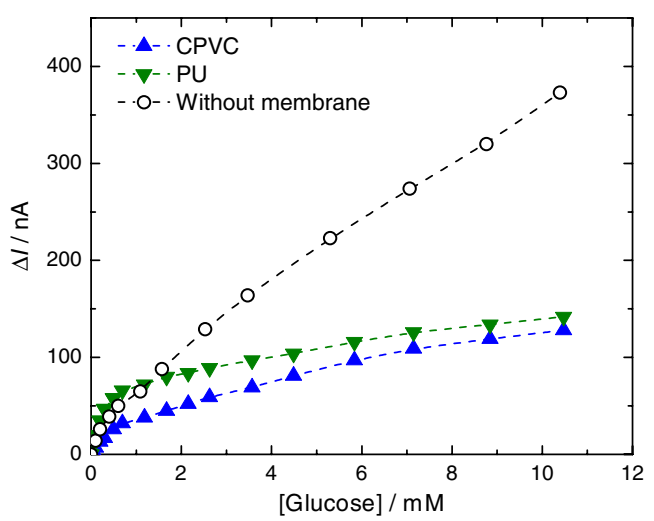

Fig. 5 Glucose calibration curves at PNR/sol-gel-GOx (open circles), $\mathrm{PNR} /$ sol-gel-GOx/CPVC (blue triangles) and PNR/sol-gel-GOx/PU (green triangles) in $0.1 \mathrm{M}$ PBS solution, $\mathrm{pH}$ 7.0. Operating potential $-0.25 \mathrm{~V}$ vs. SCE 
Table 4 Parameters calculated from calibration curves at carbon film electrodes modified with PNR as a mediator and sol-gel-encapsulated glucose oxidase with or without outer diffusion membrane $(n=3)$

\begin{tabular}{llllll}
\hline Biosensor composition & $\begin{array}{l}\text { Linear range } \\
(\mathrm{mM})\end{array}$ & $\begin{array}{l}\text { Sensitivity } \\
\left.(\mathrm{nA} \mathrm{mM})^{-1}\right)\end{array}$ & $\begin{array}{l}\text { Limit of detection } \\
(\mu \mathrm{M})\end{array}$ & $\begin{array}{l}K_{\mathrm{M}} \\
(\mathrm{mM})\end{array}$ & $\begin{array}{l}\text { Correlation coefficient } \\
\left(R^{2}\right)\end{array}$ \\
\hline PNR/sol-gel-GOx & $0.05-0.60$ & $74.7 \pm 2.8$ & 21.1 & 1.4 & 0.992 \\
PNR/sol-gel-GOx/CPVC & $0.05-0.50$ & $65.7 \pm 5.3$ & 58.2 & 1.6 & 0.997 \\
PNR/sol-gel-GOx/CPVC-IPM & $0.05-0.50$ & $52.0 \pm 2.9$ & 68.0 & 1.6 & 0.991 \\
PNR/sol-gel-GOx/PU & $0.05-0.50$ & $116.9 \pm 14.9$ & 15.8 & 1.7 & 0.997 \\
PNR/sol-gel-GOx/PU-IPM & $0.05-0.50$ & $57.0 \pm 5.8$ & 62.6 & 1.6 & 0.990 \\
\hline
\end{tabular}

All conditions as in Fig. 5

as a diffusion membrane the sensitivity in the region of the linear range appeared marginally higher than that at uncovered sol-gel. The biosensor without outer membrane had a much higher response at higher glucose concentration, which is probably related to the PNR mediation mechanism as well as some saturation of analyte diffusion through the membranes.

When IPM was used as membrane plasticizer, the response to glucose was slightly lower than that without any plasticizer (not shown) and Aliquat suppressed the signal too much (not shown), so it was not used for further investigations. Data calculated from the calibration curves are given in Table 4. The lowest limit of detection (LOD) was obtained at biosensors with the PU outer membrane but without any plasticizer. This is possibly the result of a thinner barrier layer in the absence of plasticiser. The apparent Michaelis-Menten constant obtained using Lineweaver-Burk linearisation was very similar in almost all cases, Table 4. Nevertheless, the highest sensitivity, as mentioned above, was found to be at the PNR/sol-gel-GOx/ PU biosensor. Thus, according to the data obtained, the best composition for the biosensor is a carbon film electrode modified with PNR and with enzyme immobilised in a solgel layer and using a 20\% (w/v) PU outer membrane.

The stability of the biosensors coated with outer membranes with and without IPM plasticizer was also studied. The biosensor was used once per day every day for the first 30 days and after this every second day. The results presented in Fig. 6 show that the most stable biosensor was the one with the PU outer membrane without any IPM. Note that the second measurement on the first day was used as reference as there was a significant response drift from the first measurement to the second, possibly due to leaching of free enzyme residues and some hydration effect of the sol-gel protein membrane [44]. The response did not drop further with PU even after 70 days. However, the biosensor with PU and plasticizer was stable for only 30 days and then the signal started to decrease progressively reaching 50\% after 70 days. When CPVC was used as the outer membrane, stability was significantly lower and at the biosensor without plasticizer the signals started to decrease after 40 days. IPM makes the sensor even less stable than sol-gel without any outer membrane. It is possible that the plasticizers here changed the membrane morphology, e.g. by altering pore geometry [45], in addition to the effect on the dielectric properties of the polymer phase; this would affect enzyme retention ability. Alternatively it could be an ageing effect of the membrane itself. The lifetime of the biosensor without any outer membrane was 40 days [40].

The storage stability of the biosensors was at least 5 months when the sensors were kept at $+4{ }^{\circ} \mathrm{C}$ in PBS and at least 1 month under dry conditions at the same temperature for biosensors with outer membranes without plasticizer and 2 months with plasticizers. The membranes began to crack after they had been stored under dry conditions for a longer period of time.

\section{Investigation of interferences}

Interference from some organic acids and some other organic compounds, such as fructose and phenol, was in-

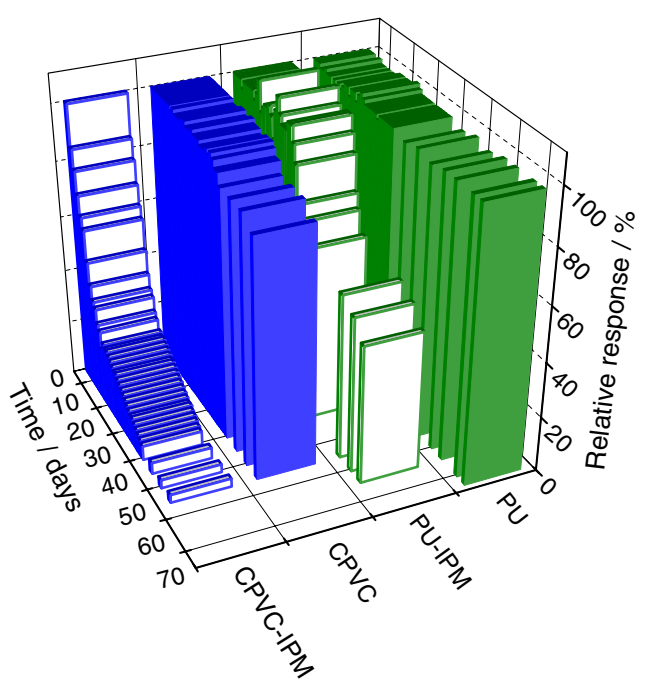

Fig. 6 Response to $0.3 \mathrm{mM}$ glucose over time relative to initial value at PNR/sol-gel-GOx electrodes covered by PU, PU-IPM, CPVC and CPVC-IPM outer membranes. All other conditions as in Fig. 5 
Table 5 Interference of some compounds on response to $0.2 \mathrm{mM}$ of glucose at PNR/sol-gel-GOx/CPVC and PNR/sol-gel-GOx/PU biosensors

\begin{tabular}{llll}
\hline Biosensor & Compound & $\begin{array}{l}\text { Glucose/interfering } \\
\text { compound ratio }\end{array}$ & $\begin{array}{l}\text { Response } \\
\text { signal (\%) }\end{array}$ \\
\hline PNR/sol-gel- & Fructose & $1: 5$ & 100.0 \\
GOx/CPVC & Phenol & $1: 5$ & 112.5 \\
& Acetaldehyde & $1: 1$ & 108.3 \\
& Acetic acid & $1: 5$ & 133.3 \\
& Ascorbic acid & $1: 5$ & 100.0 \\
& Citric acid & $1: 1$ & 97.9 \\
& & $1: 5$ & 100 \\
PNR/sol-gel- & Tartaric acid & $1: 1$ & 75.6 \\
GOx/PU & Fructose & $1: 5$ & 92.8 \\
& Phenol & $1: 5$ & 75.6 \\
& Acetaldehyde & $1: 1$ & 100.0 \\
& $1: 5$ & 111.1 \\
& & 129.6 \\
& Acetic acid & $1: 5$ & 85.7 \\
& Ascorbic acid & $1: 5$ & 71.4 \\
& Citric acid & $1: 5$ & 100.0 \\
& Tartaric acid & $1: 1$ & 91.7 \\
& $1: 5$ & 100.0 \\
& & 100.0 \\
\hline
\end{tabular}

All conditions as in Fig. 5

vestigated at biosensors with both CPVC and PU outer membranes, without any plasticizer. Most organic acids only interfered at much higher concentrations than glucose (Table 5). Fructose hardly interferes, but quite a significant interference was found from phenol, which increased the glucose signal. The interferences are lower when a PU membrane is used, except from acetaldehyde. This is possibly related to the higher concentration of PU compared with CPVC so that a more homogeneous PU membrane is formed (see Scanning electron microscopy) which better prevents penetration of an interfering compound. The interferents react with PNR, which easily reacts electrochemically with compounds having carboxylic or alcohol groups [14, 37, 38, 46]. On the other hand, after application of the outer membranes the interferences decreased significantly compared with other biosensors based on PNR mediators [38], which confirms that these membranes, especially PU, hinder access of interferent to the mediator layer. The much reduced interference also confirms the relatively defect-free PU membranes produced.

\section{Application to natural samples}

The biosensors were applied to glucose determination in a commercially available orange drink, which consists of $10 \%(\mathrm{v} / \mathrm{v})$ natural orange juice and either sugar syrup or a glucose/fructose mixture, or both. Biosensors with both outer membranes (CPVC or PU) were employed for glucose determination in the orange drink by the standard addition method. The sample was diluted 100 times to be within the biosensor linear range. Concentrations of $0.229 \pm$ $0.011 \mathrm{M}\left(41.2 \pm 2.0 \mathrm{~g} \mathrm{~L}^{-1}\right)$ and $0.204 \pm 0.026 \mathrm{M}(36.8 \pm$ $4.7 \mathrm{~g} \mathrm{~L}^{-1}$ ) of glucose were found at biosensors with CPVC and PU outer membranes, respectively. The PNR/sol-gelGOx/PU biosensor showed a lower value and a higher uncertainty than the PNR/sol-gel-GOx/CPVC one. However, these values are in a good agreement with the value obtained by the spectrophotometric reference method using an enzymatic test kit according to the procedure described elsewhere [47]: $0.214 \pm 0.005 \mathrm{M}\left(38.6 \pm 0.8 \mathrm{~g} \mathrm{~L}^{-1}\right)$.

\section{Conclusions}

The performance of sol-gel-based biosensors was improved by application of an outer protecting polymer membrane, particularly carboxylated PVC and polyurethane. Three types of membrane composition were investigated: polymer without any plasticizer and with two different plasticizers, Aliquat-336 and IPM. These biosensors were first characterised electrochemically, using CV and EIS. Sol-gel and protecting membrane layers decreased the PNR re-oxidation current since they act as diffusion membranes. The membranes decrease interferences significantly, and extend the lifetime of the biosensor to more than 2.5 months, especially in the case when PU without any plasticizer is used as outer membrane. The membranecoated biosensor with PU outer membrane also exhibited higher sensitivity and a lower limit of detection. This biosensor construction, i.e. with sol-gel enzyme layer covered by a polyurethane membrane, is recommended for future studies and applications.

Acknowledgements The financial support of the Treaty of Windsor Anglo-Portuguese Joint Research Programme 2005-6, of ICEMSCoimbra (Research Unit 103) Portugal, and of the Biotechnology and Biological Sciences Research Council, UK, is gratefully acknowledged. R. Pauliukaite thanks Fundação para a Ciência e Tecnologia (FCT) for a postdoctoral grant (SFRH/BPD/14518/2003).

\section{References}

1. Gill I, Ballesteros A (1998) J Am Chem Soc 120:8587

2. Gill I (2001) Chem Mater 13:3404

3. Pierre AC (2004) Biocatal Biotransform 22:145

4. Walcarius A, Mandler D, Cox JA, Collinson M, Lev O (2005) J Mater Chem 15:3663

5. Tripathi VS, Kandimalla VB, Ju H (2006) Sens Actuat B 114:1071 
6. Anitha S, Mohan SV, Reddy SJ (2004) Biosens Bioelectron 20:848

7. Lei CX, Hu SQ, Gao N, Shen GL, Yu RQ (2004) Bioelectrochem 65:33

8. Roman G, Pappas ACh, Kovala-Demertzi D, Prodromidis MI (2004) Anal Chim Acta 523:201

9. Salimi A, Compton RG, Hallaj R (2004) Anal Biochem 333:49

10. Teh HF, Yang X, Gong H, Tan SN (2004) Electroanalysis 16:769

11. Xu JZ, Zhang Y, Li GX, Zhu JJ (2004) Mater Sci Eng C 24:833

12. Tu YF, Di JW, Chen XJ (2005) J Sol-Gel Sci Technol 33:187

13. Pauliukaite R, Brett CMA (2005) Electrochim Acta 50:4973

14. Pauliukaite R, Brett CMA, Chiorcea-Paquim AM, Oliveira Brett AM (2006) Electrochim Acta 52:1

15. Oh BK, Robbins ME, Nablo BJ, Schoenfisch MH (2005) Biosens Bioelectron 21:749

16. Pastor I, Esquembre R, Micol V, Mallavia R, Reyes Mateo C (2004) Anal Biochem 334:335

17. Wu HJ, Choi MMF (2004) Anal Chim Acta 514:219

18. Wang J (1999) Anal Chem 71:328R

19. Gill I, Ballesteros A (2000) Trends in Biotechnol 18:469

20. Wisniewski N, Reichert M (2000) Coll Surf 18:197

21. Reddy SM, Vadgama P (2002) Anal Chim Acta 461:57

22. Maines A, Ashworth D, Vadgama P (1996) Anal Chim Acta 333:223

23. Puig-Lleixa C, Jiménez C, Bartroli J (2001) Sens Actuat B $72: 56$

24. Vastarella W, Nicastri R (2005) Talanta 66:627

25. Choi SH, Lee SD, Shin JH, Ha J, Nam H, Cha GS (2002) Anal Chim Acta 461:251

26. Lau RCW, Choi MHF, Lu J (1999) Talanta 48:321

27. Koncki R, Kopczewska E, Glab S (1994) Anal Lett 27:475
28. Karakuș E, Pekyardımcı Ş, Kılıç E (2005) Art Cell Blood Subst Biotechnol 33:329

29. Brett CMA, Angnes L, Liess H-D (2001) Electroanalysis 13:765

30. Filipe OMS, Brett CMA (2003) Talanta 61:643

31. Filipe OMS, Brett CMA (2004) Electroanalysis 16:994

32. Florescu M, Brett CMA (2005) Talanta 65:306

33. Florescu M, Brett CMA (2004) Anal Lett 37:871

34. Ghica ME, Brett CMA (2005) Anal Chim Acta 532:145

35. Pauliukaite R, Ghica ME, Barsan M, Brett CMA (2007) J Solid State Electrochem 11:899

36. Ferrer ML, del Monte F, Levy D (2002) Chem Mater 14:3619

37. Karyakin AA, Bobrova OA, Karyakina EE (1995) J Electroanal Chem 399:179

38. Ghica ME, Brett CMA (2006) Electroanalysis 18:748

39. de Jesus DS, Couto CMCM, Araujo AN, Montenegro MCBSM (2003) J Pharm Biomed Anal 33:983

40. Chiorcea Paquim AM, Pauliukaite R, Brett CMA, Oliveira Brett AM In preparation

41. Buenfeld NR, Zhang JZ (2000) J Mater Sci 35:39

42. Khobaib M, Reynolds LB, Donley MS (2001) Surf Coat Technol 140:16

43. Orazem ME, Shukla P, Membrino MA (2002) Electrochim Acta 47:2027

44. Chen JY, Cox W, Tehan E, Bright FV, Cerne J, Markelz AG (2004) Can be downloaded online at http://arxiv.org/ftp/cond-mat/ papers/0307/0307558.pdf

45. Tan K, Obendorf SK (2006) J Memb Sci 274:150

46. Broncova G, Shishkanova TV, Matejka P, Volf R, Kral V (2004) Anal Chim Acta 511:197

47. Bergmeyer HU, Bernt E, Schmidt F, Stork H (1974) Methoden der enzymatischen Analyse, 3rd edn, Vol 2. Verlag Chemie, Weinheim 